\title{
REDUCTION OF KNOWLEDGE VIA MINIMAL APPROXIMATION SPACE AND GRAPHS
}

\author{
M. M. El-Sharkasy ${ }^{1}$ \\ 1- Department of Mathematics, Faculty of Science, Tanta University, Tanta, Egypt. \\ E-mail: sharkasy78@yahoo.com \& mohamed.elsharkasi@science.tanta.edu.eg.
}
Received 18/3/2018
Revised 31/3/2018
Accepted 25/5/2018

\begin{abstract}
In this paper, we introduce a simple undirected graph based on a binary relation by using the notion of right neighborhood and minimal approximation space. Also, we investigate the lower approximation and the upper approximation of a subgraph to compute the rough subgraph. Finally, we will make a reduction of an information system and compute the core of knowledge by using a minimal approximation space and a graph theory.
\end{abstract}

Keywords: Approximation space, graph theory, information system, minimal structure and rough sets.

Mathematics subject classification: 54A05, 54C55, 54E05, 57M07

\section{Introduction and Preliminaries.}

In 1736 graph theory has been introduced by Euler [1], which considered the (general case of the) Konigsberg bridge problem. Graphs are an important discrete structure consisting of vertices and edges that connect these vertices and they describe the relationships among objects $[2,3,4,5]$. The notion of minimal structure has been introduced by V. Popa and T. Noiri [6]. Rough set theory introduced by Pawlak [7], the relation between rough set and topological spaces, data-mining and graph theory studied in $[8,9,10,11,12,13,14]$.

In this paper, we use the notion of the right neighborhood and minimal structure to introduce a simple undirected graph based on a binary relation. Also, we compute the lower approximation, the upper approximation and the rough subgraph. Finally, we use the minimal approximation space and graph theory to reduct the information system and computing the core of knowledge.

Definition 1.1. [3] A graph $G$ is a pair $\left(V_{G}, E_{G}\right)$ where $V_{G}$ is the set vertices and $E_{G}$ is the edges between vertices.

A graph may be directed graphs or undirected graphs [3] if the edges have a direction or no direction.

Definition 1.2. [3] A graph $G$ is called simple if every edge links a unique pair of distinct vertices.

Definition 1.3. [2] The neighbourhood set of $v \in V_{G}$ is $N_{G}(v)=\left\{u \in V_{G}: v u \in E_{G}\right\}$ and the degree of $v$ is the number of elements of its neighbourhood $d_{G}(V)=\left|N_{G}(V)\right|$.

If $d_{G}(v)=0$, then $v$ is said to be isolated in $G$, and if $d_{G}(v)=1$, then $v$ is a leaf of the graph. The minimum degree and the maximum degree of $G$ are denoted as:

$\operatorname{Min}(G)=\min \left\{d_{G}(v): v \in V_{G}\right\}$ and $\operatorname{Max}(G)=\max \left\{d_{G}(v): v \in V_{G}\right\}$

Definition 1.4. [3] A graph $H$ is a subgraph of $G$, denoted by $H \subseteq G$ if $V_{H} \subseteq V_{G}$ and $E_{H} \subseteq E_{G}$. A subgraph $H$ spans $G$ ( $H$ is spanning subgraph of $G$ ) if $V_{H}=V_{G}$. 
Definition 1.5. [14] Let $G(U, E)$ be a graph, a subset $X$ of $U$ called an independent set of $G$ for any $x \in$ $X$, there does not exist $y \in X$ such that $(x, y) \in R$. Hence we obtain

$$
R_{s}(x) \cap X=\emptyset, \forall x \in X .
$$

An independent set is a set of pairwise nonadjacent vertices.

Definition 1.6. [14] A graph $G$ is called bipartite if the vertex set $U$ can to be partitioned into two sets $X$ and $Y$ such that each edge has one end in $X$ and one end in $Y$.

Definition 1.7. [11] Let $(U, R)$ be a generalized approximation space where $\mathrm{U}$ be non-empty set and $R$ be a binary relation on $U$. The set $N(x)=\{y \in U: x R y\}$ is the right neighborhood of $x$ for all $x \in U$ and the class $M S(U)=\{\phi, U, N(x)$ for all $x \in U\}$ is called a minimal structure on $(U, R)$.

Definition 1.8. [7] A family set $S=(U, A, V, F)$ is called an information system (IS), where $U$ is a nonempty finite set of objects, $A$ is a nonempty finite set of attributes and $V$ is the union of attribute domains and $F: \mathrm{U} \times A \rightarrow V$ is an information function which associates a unique value of each attribute with every object belong to $U$.

\section{Methods of generating graphs of binary relation by minimal approximation space.}

In this section, we will introduce undirected graph of binary relations by using right neighborhoods. Also, we introduce the lower approximation and upper approximation of subgraph by using the minimal structure approximation space to the rough graph.

Definition 2.1. Let $(U, R, M S)$ be minimal structure approximation space. A graph $\mathrm{G}$ can be defined by considering the set $U$ is the set of all vertices and the edges between the two vertices $\mathrm{x}$ and $\mathrm{y}$ in $\mathrm{U}(x e y)$ defined by $x e y$ if $N(x) \cap N(y) \neq \varnothing$.

Example 2.1. Let $U=\{a, b, c, d\}$ and $R=\{(a, a),(a, b),(b, a),(a, c),(c, a),(b, c),(c, b),(c, d),(d, c)\}$. We observe that $R$ is a general binary relation. Then $N(a)=\{a, b, c\}, N(b)=\{a, c\}, N(c)=\{a, b, d\}, N(d)=\{c\}$, and the graph of $R$ is shown as in Figure 1.

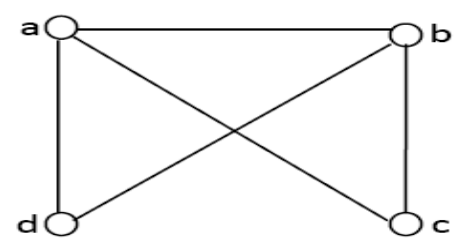

Figure 1

Example 2.2. Let $U=\{a, b, c, d\}$ and $R=\{(a, a),(a, b),(a, c),(a, d),(b, a),(b, b)$, $(b, c),(c, a),(c, b),(c, c),(c, d),(d, a),(d, c),(d, d)\}$. Then $N(a)=U, N(b)=\{a, b, c\}, N(c)=U, N(d)=\{a, c, d\}$, and the graph of $R$ is shown as in Figure 2.

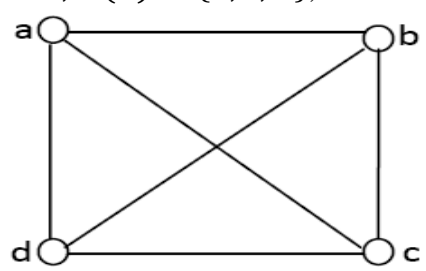

Figure 2

Definition 2.2. Let $(U, R, M S)$ be MSAS and $H$ with vertex set $\mathrm{V}(\mathrm{H})$ is a subgraph of a simple undirected graph $G$. Then the minimal lower approximation, the minimal upper approximation, and minimal boundary define as follows:
1- $\underline{M S}(\mathrm{~V}(\mathrm{H}))=\mathrm{U}\{\mathrm{A} \in \mathrm{MS}: \mathrm{A} \subseteq \mathrm{V}(\mathrm{H})\}$;
2- $\overline{M S}(\mathrm{~V}(\mathrm{H}))=\mathrm{n}\left\{\mathrm{F} \in(\mathrm{MS})^{\mathrm{C}}: \mathrm{V}(\mathrm{H}) \subseteq \mathrm{F}\right\}$; 
3- $M S-B N(V(H))=\overline{M S}(\mathrm{~V}(\mathrm{H}))-\underline{M S}(\mathrm{~V}(\mathrm{H}))$.

$\mathrm{V}(\mathrm{H})$ is $M S$ - exact if and only if $\underline{M S}(\mathrm{~V}(\mathrm{H}))=\overline{M S}(\mathrm{~V}(\mathrm{H}))$ otherwise $\mathrm{V}(\mathrm{H})$ is $M S$-rough. i.e., $\mathrm{H}$ is exact subgraph of graph $\mathrm{G}$ if $M S$ - $\mathrm{BN}(\mathrm{V}(\mathrm{H}))=\varnothing$ otherwise $\mathrm{H}$ is rough.

Definition 2.3. The accuracy measure of a subgraph $\mathrm{H}$ can be defined as $\mu(V(H))=\frac{|\underline{M S}(\mathrm{~V}(\mathrm{H}))|}{|\overline{M S}(\mathrm{~V}(\mathrm{H}))|}$, where $\mid$ denotes cardinality of the set $V(H)$ and $V(H) \neq \varnothing$ and $0 \leq$ $\mu(V(H)) \leq 1$. If $\mu(V(H))=1$, then $(V(H))$ is definable.

Example 2.3. From Example 2.1, we have $M S(X)=\{\varnothing, U,\{a, b, c\},\{a, c\},\{a, b, d\},\{c\}\}$ and the accuracy measures of subgraph $H$ are calculated as in Table 1.

\begin{tabular}{|c|c|c|c|}
\hline $\mathrm{V}(\mathrm{H})$ & $\underline{M S}(\mathrm{~V}(\mathrm{H}))$ & $\overline{\boldsymbol{M S}}(\mathrm{V}(\mathrm{H}))$ & $\mu(M S(V(H)))$ \\
\hline$\{a\}$ & $\varnothing$ & $\{a, b, d\}$ & 0 \\
\hline$\{b\}$ & $\varnothing$ & $\{b, d\}$ & 0 \\
\hline$\{c\}$ & $\{c\}$ & $\{c\}$ & 1 \\
\hline$\{d\}$ & $\varnothing$ & $\{d\}$ & 0 \\
\hline$\{a, b\}$ & $\varnothing$ & $\{a, b, d\}$ & 0 \\
\hline$\{a, c\}$ & $\{a, c\}$ & $\{a, b, d\}$ & 0 \\
\hline$\{a, d\}$ & $\varnothing$ & $U$ & $1 / 4$ \\
\hline$\{b, c\}$ & $\{c\}$ & $\{b, d\}$ & 0 \\
\hline$\{b, d\}$ & $\varnothing$ & $U$ & $1 / 4$ \\
\hline$\{c, d\}$ & $\{c\}$ & $U$ & $3 / 4$ \\
\hline$\{a, b, c\}$ & $\{a, b, c\}$ & $\{a, b, d\}$ & 1 \\
\hline$\{a, b, d\}$ & $\{a, b, d\}$ & $U$ & $2 / 4$ \\
\hline$\{a, c, d\}$ & $\{a, c\}$ & $U$ & $2 / 4$ \\
\hline$\{b, c, d\}$ & $\{c\}$ & & \\
\hline
\end{tabular}

Table 1

From Table 1, we notice that the exact subgraph $H=\{a, b, d\}$ or $H=\{c\}$ and the other subgraph is rough.

Proposition 2.1. Let $H$ be a subgraph of simple undirected graph $G$. Then $\underline{M S}(\mathrm{~V}(\mathrm{H})) \subseteq \mathrm{V}(\mathrm{H}) \subseteq$ $\overline{M S}(\mathrm{~V}(\mathrm{H}))$.

Proof. From Definition 2.2. Since $\mathrm{V}(\mathrm{H}) \subseteq \overline{M S}(\mathrm{~V}(\mathrm{H}))$ and $\underline{M S}(\mathrm{~V}(\mathrm{H})) \subseteq \mathrm{V}(\mathrm{H})$, then $\underline{M S}(\mathrm{~V}(\mathrm{H})) \subseteq$ $\mathrm{V}(\mathrm{H}) \subseteq \overline{M S}(\mathrm{~V}(\mathrm{H}))$.

Proposition 2.2. The minimal lower approximation and minimal upper approximation of a simple undirected graph $G$ (null graph) are equal to the vertices of $G$ (itself).

Proof. The proof is direct from the Definition 2.2 and Proposition 2.1.

Example 2.4. If $Q$ is the null graph then $\underline{M S}(Q) \subseteq \emptyset \subseteq \overline{M S}(\mathrm{Q})$ and $\underline{M S}(\mathrm{~V}(\mathrm{G})) \subseteq \mathrm{V}(\mathrm{G})=\mathrm{U} \subseteq$ $\overline{M S}(\mathrm{~V}(\mathrm{G}))$.

Proposition 2.3. For any two subgraphs $\mathrm{V}(\mathrm{Q}), \mathrm{V}(\mathrm{W})$ of a simple undirected graph $G$. We have:

1. If $\mathrm{V}(\mathrm{Q}) \subseteq \mathrm{V}(\mathrm{W})$, then $\overline{M S}(\mathrm{~V}(\mathrm{Q})) \subseteq \overline{M S}(\mathrm{~V}(\mathrm{~W}))$;

2. If $\mathrm{V}(\mathrm{Q}) \subseteq \mathrm{V}(\mathrm{W})$, then $\underline{M S}(\mathrm{~V}(\mathrm{Q})) \subseteq \overline{M S}(\mathrm{~V}(\mathrm{~W}))$;

3. $\overline{M S}(\mathrm{~V}(\mathrm{Q})) \cup \overline{M S}(\mathrm{~V}(\mathrm{~W})) \subseteq \overline{M S}(\mathrm{~V}(\mathrm{Q} \cup \mathrm{W}))$;

4. $\overline{M S}(\mathrm{~V}(\mathrm{Q} \cap \mathrm{W})) \subseteq \overline{M S}(\mathrm{~V}(\mathrm{Q})) \cap \overline{M S}(\mathrm{~V}(\mathrm{~W}))$;

5. $\underline{M S}(\mathrm{~V}(\mathrm{Q})) \cup \underline{M S}(\mathrm{~V}(\mathrm{~W})) \subseteq \underline{M S}(\mathrm{~V}(\mathrm{Q} \cup \mathrm{W}))$;

6. $\underline{M S}(\mathrm{~V}(\mathrm{Q} \cap \mathrm{W})) \subseteq \underline{M S}(\mathrm{~V}(\mathrm{Q})) \cap \underline{M S}(\mathrm{~V}(\mathrm{~W}))$. 
Proof. Obvious.

Example 2.5. From Example 2.3. If :-

1- $\mathrm{V}(\mathrm{Q})=\{b\}, \mathrm{V}(\mathrm{W})=\{c\}$, then $\overline{M S}(\mathrm{~V}(\mathrm{Q})) \cup \overline{M S}(\mathrm{~V}(\mathrm{~W}))=\{\mathrm{b}, \mathrm{c}, \mathrm{d}\} \subseteq \mathrm{U}=\overline{M S}(\mathrm{~V}(\mathrm{Q} \cup \mathrm{W}))$

2- $\mathrm{V}(\mathrm{Q})=\{a\}, \mathrm{V}(\mathrm{W})=\{c\}$, then $\underline{M S}(\mathrm{~V}(\mathrm{Q})) \cup \underline{M S}(\mathrm{~V}(\mathrm{~W}))=\{\mathrm{c}\} \subseteq\{\mathrm{a}, \mathrm{c}\}=\underline{M S}(\mathrm{~V}(\mathrm{QUW}))$.

3- $\mathrm{V}(\mathrm{Q})=\{a\}, \mathrm{V}(\mathrm{W})=\{b\}$, then $\overline{M S}(\mathrm{~V}(\mathrm{Q} \cap \mathrm{W}))=\emptyset \subseteq\{\mathrm{b}, \mathrm{d}\}=\overline{M S}(\mathrm{~V}(\mathrm{Q})) \cap \overline{M S}(\mathrm{~V}(\mathrm{~W}))$.

4- $\mathrm{V}(\mathrm{Q})=\{a, b, c\}, \mathrm{V}(\mathrm{W})=\{a, b, d\}$, then $\underline{M S}(\mathrm{~V}(\mathrm{Q} \cap \mathrm{W}))=\emptyset \subseteq\{\mathrm{a} \cdot \mathrm{b}\}=\underline{M S}(\mathrm{~V}(\mathrm{Q})) \cap$ $\underline{M S}(\mathrm{~V}(\mathrm{~W}))$.

Definition 2.4. Let $(U, R, M S)$ be MSAS, for any subgraph $H$ with vertex set $\mathrm{V}(\mathrm{H})$ of a simple undirected graph $G$. $H$ is said to be:

1- Outer definable set (resp. Quasi-outer definable set) if $\overline{M S}(H)=H($ resp. $\overline{M S}(\overline{M S}(H))=H)$. The family of outer definable sets (resp. quasi-outer definable sets) is denoted by $\Omega(U, R, M S)$ (resp. $\mu(U, R, M S)$;

2- Inner definable set (resp. quasi-Inner definable set) if $\underline{M S}(H)=H(\operatorname{resp} . \underline{M S}(\underline{M S}(H))=H)$. The family of all Inner definable sets (resp. Quasi-Inner definable sets) is denoted by $\gamma(U, R, M S)($ resp. $\psi(U, R, M S))$.

Example 2.6. From Example 2.1, we have:

$\Omega(U, R, M S)=\psi(U, R, M S)=\{\varnothing, U,\{a, b, c\},\{a, c\},\{a, b, d\},\{c\}\}$ and $\partial(U, R, M S)=\mu(U, R, M S)=\{\varnothing, U,\{d\},\{c\},\{b, d\},\{a, b, d\}\}$.

Remark 2.2. The class of Outer definable contains the class of minimal closed set and the class of inner definable set contains the class of minimal open set but the converse is not true as in Example 2.7 .

Example 2.7. Let $U=\{a, b, c, d\}$ and $R=\{(a, a),(b, b)\}$. Then

$\operatorname{MS}(X)=\{\varnothing, U,\{a\},\{b\}\}$,

$M S^{c}(X)=\{\varnothing, U,\{b, c, d\},\{a, c, d\}\}$,

$\Omega(U, R, M S)=\psi(U, R, M S)=\{\varnothing, U,\{a\},\{a, b\},\{b\}\}$ and

$\partial(U, R, M S)=\mu(U, R, M S)=\{\varnothing, U,\{b, c, d\},\{a, c, d\},\{c . d\}\}$.

Proposition 2.4. Let $(U, R, M S)$ be a MSAS. Then:

1- $\partial(U, R, M S)=\mu(U, R, M S)$;

2- $\quad \Omega(U, R, M S)=\psi(U, R, M S)$.

\section{Proof.}

1- For any $H \in \partial(U, R, M S)$ we have $\overline{M S}(H)=H$. Then by Definition 2.2, we have $\overline{M S}(\overline{M S}(H))=\overline{M S}(H)=H$. Therefore $\partial(U, R, M S)=\mu(U, R, M S)$.

2- Let $H \in \Omega(U, R, M S)$. Then $\underline{M S}(H)=H$. Then by Definition 2.2, we have and $\underline{M S}(\underline{M S}(H))=\underline{M S}(H)=H$. Therefore $\Omega(U, R, M S)=\psi(U, R, M S)$.

Remark 2.1. J.Chen and J.Li [14] define the outer definable set, inner definable set, quasi-outer definable set and quasi-inner definable set on serial relations. But, we define these sets on general relations as in Definition 2.4. Also, we observe the class of outer definable set equal to the class of quasi-outer definable set and the class of inner definable set is equal to the class of quasi-inner definable set as in Proposition 2.4 .

Proposition 2.5. Let $(U, R, M S)$ be a MSAS and $H, H_{1}, H_{2} \in \mu(U, R, M S)$. Then:

1- $H_{1} \cup H_{2} \in \mu(U, R, M S)$;

2- $H_{1} \cap H_{2} \in \mu(U, R, M S)$.

Proof.

1- Let $H_{1}, H_{2} \in \mu(U, R, M S)$. Then $H_{1} \cup H_{2} \subseteq \overline{M S}\left(\overline{M S}\left(H_{1} \cup H_{2}\right)\right) \rightarrow$ (1). 
On the other hand, let $h \notin H_{1} \cup H_{2}=\overline{M S}\left(\overline{M S}\left(H_{1}\right)\right) \cup \overline{M S}\left(\overline{M S}\left(H_{1}\right)\right) \Rightarrow$ $h \notin \overline{M S}\left(\overline{M S}\left(H_{1} \cup H_{2}\right)\right)$. Then $\overline{M S}\left(\overline{M S}\left(H_{1} \cup H_{2}\right)\right) \subseteq H_{1} \cup H_{2} \rightarrow$ (2).

From 1 and 2 we have $H_{1} \cup H_{2}=\overline{M S}\left(\overline{M S}\left(H_{1} \cup H_{2}\right)\right)$ and

$$
H_{1} \cup H_{2} \in \mu(U, R, M S) \text {. }
$$

2- Let $H_{1}, H_{2} \in \mu(U, R, M S)$. Then $H_{1} \cap H_{2} \subseteq \overline{M S}\left(\overline{M S}\left(H_{1} \cap H_{2}\right)\right) \subseteq \overline{M S}\left(\overline{M S}\left(H_{1}\right)\right) \cap$

$$
\begin{aligned}
& \overline{M S}\left(\overline{M S}\left(H_{1}\right)\right)=H_{1} \cap H_{2} \text {. Then } \\
& H_{1} \cap H_{2}=\overline{M S}\left(\overline{M S}\left(H_{1} \cap H_{2}\right)\right) \text { and } H_{1} \cap H_{2} \in \mu(U, R, M S) .
\end{aligned}
$$

Proposition 2.6. Let $(U, R, M S)$ be MSAS. For $H, H_{1}, H_{2} \in \Psi(U, R, M S)$. Then:

1- $H_{1} \cup H_{2} \in \Psi(U, R, M S)$.

2- $H \cap H_{2} \in \psi(U, R, M S)$.

Proof. Similar to the proof of Proposition 2.5.

\section{3- Reduction of Knowledge and core of Knowledge.}

In this section, we will do the reduction of knowledge of information system by two methods one of them by using the notion of the quality of approximation of classification and the other method is by using the graph of information system.

Definition 3.1. Let $S=(U, \mathcal{A}, V, F)$ be an information system. The quality of approximation of classification $\gamma_{R}(X)=\frac{\operatorname{Card}\left(P O S_{R}(X)\right)}{\operatorname{Card}(U)}$, where $\left.\operatorname{POS}_{R}(X)=\bigcup \underline{U S_{\mathcal{A}}(u)}: u \in M S_{a}(U)\right\}$.

Example 3.1. Considering the information system $S=(U, \mathcal{A}, V, F)$ as in Table 2. Where the set of objects $U=\left\{x_{1}, x_{2}, x_{3}, x_{4}, x_{5}\right\}$ represent the ID of 5 students and the set of attributes $\mathcal{A}=\{A, E, M, C\}$ are the degrees scored by students in an examination.

\begin{tabular}{|l|l|l|l|l|}
\hline & $A$ & $E$ & $M$ & $C$ \\
\hline$x_{1}$ & 90 & 85 & 71 & 95 \\
\hline$x_{2}$ & 85 & 64 & 65 & 83 \\
\hline$x_{3}$ & 74 & 69 & 83 & 92 \\
\hline$x_{4}$ & 86 & 88 & 92 & 64 \\
\hline$x_{5}$ & 72 & 95 & 81 & 70 \\
\hline \multicolumn{5}{|c}{ Table 2 }
\end{tabular}

Consider the relation $\mathrm{R}$ on the set objects defined by $x_{i} R x_{j} \Leftrightarrow\left|a\left(x_{i}\right)-a\left(x_{j}\right)\right|<5$.

For $\mathcal{A}=(A, E, M, C)$,

\begin{tabular}{|c|c|c|c|c|c|}
\hline$(A+E+M+C) / 4$ & $x_{1}$ & $x_{2}$ & $x_{3}$ & $x_{4}$ & $x_{5}$ \\
\hline$x_{1}$ & 0 & 11 & 5,75 & 2,75 & 5,75 \\
\hline$x_{2}$ & 11 & 0 & 5,25 & 8,25 & 5,25 \\
\hline$x_{3}$ & 5,75 & 5,25 & 0 & 3 & 0 \\
\hline$x_{4}$ & 2,75 & 8,25 & 3 & 0 & 3 \\
\hline$x_{5}$ & 5,75 & 5,25 & 0 & 3 & 0 \\
\hline
\end{tabular}

Table 4

From Table 4, we have

$M S_{\mathcal{A}}(U)=\left\{\varnothing, U,\left\{x_{1}, x_{4}\right\},\left\{x_{2}\right\},\left\{x_{3}, x_{4}, x_{5}\right\},\left\{x_{1}, x_{3}, x_{4}, x_{5}\right\},\left\{x_{3}, x_{4}, x_{5}\right\}\right\}$ and

$\gamma_{\mathcal{A}}(X)=1$.

For attribute $A$,

\begin{tabular}{|c|l|l|l|l|l|}
\hline$A$ & $x_{1}$ & $x_{2}$ & $x_{3}$ & $x_{4}$ & $x_{5}$ \\
\hline$x_{1}$ & 0 & 5 & 16 & 4 & 18 \\
\hline$x_{2}$ & 5 & 0 & 11 & 1 & 13 \\
\hline
\end{tabular}




\begin{tabular}{|l|l|l|l|l|l|}
\hline$x_{3}$ & 16 & 11 & 0 & 12 & 2 \\
\hline$x_{4}$ & 4 & 1 & 12 & 0 & 1 \\
\hline$x_{5}$ & 18 & 13 & 2 & 14 & 0 \\
\hline
\end{tabular}

Table 3

From Table 3, we have $N\left(x_{1}\right)=\left\{x_{1}, x_{4}\right\}, N\left(x_{2}\right)=\left\{x_{2}, x_{4}\right\}, N\left(x_{3}\right)=\left\{x_{3}, x_{5}\right\}$,

$N\left(x_{4}\right)=\left\{x_{1}, x_{2}, x_{4}\right\}$ and $N\left(x_{5}\right)=\left\{x_{3}, x_{5}\right\}$. Then

$M S_{A}(U)=\left\{\varnothing, U,\left\{x_{1}, x_{4}\right\},\left\{x_{2}, x_{4}\right\},\left\{x_{3}, x_{5}\right\},\left\{x_{1}, x_{2}, x_{4}\right\},\left\{x_{3}, x_{5}\right\}\right\}$ and

$\gamma_{A}(X)=\frac{4}{5} \neq \gamma_{\mathcal{A}}(X)$.

By similar way we have $x_{i} R_{(A+E) / 2}=\left\{\left\{x_{1}, x_{4}, x_{5}\right\},\left\{x_{2}, x_{3}\right\}\right\}$ and

$M S_{(A+E) / 2}(U)=\left\{\varnothing, U,\left\{x_{1}, x_{4}, x_{5}\right\},\left\{x_{2}, x_{3}\right\}\right\}$.

Then $\operatorname{POS}_{(A+E) / 2}(X)=\left\{x_{1}, x_{4}, x_{5}\right\}$ and $\gamma_{R_{(A+E) / 2}}(X)=\frac{3}{5} \neq \gamma_{M_{S}}(X)$.

Also, we have $x_{i} R_{(A+E+M) / 3}=\left\{\left\{x_{1}, x_{5}\right\},\left\{x_{2}, x_{3}\right\},\left\{x_{4}\right\}\right\}$ and

$M S_{(A+E) / 2}(U)=\left\{U, \emptyset,\left\{x_{1}, x_{5}\right\},\left\{x_{2}, x_{3}\right\},\left\{x_{4}\right\}\right\}$

Then $P O S_{R_{(A+E+M) / 3}}(X)=\left\{x_{1}, x_{4}, x_{5}\right\}$ and $\gamma_{R_{(A+E+M) / 3}}(X)=\frac{3}{5} \neq \gamma_{M_{S}}(X)$.

Then by the similar way the quality of approximation of classification of all subsets of attribute can be obtained as in Table 5.

\begin{tabular}{|c|c|c|}
\hline & $P O S(X)$ & $\gamma$ \\
\hline$A$ & $\left\{x_{1}, x_{2}, x_{4}\right\}$ & $3 / 5$ \\
\hline$E$ & $\left\{x_{1}, x_{2}, x_{4}\right\}$ & $3 / 5$ \\
\hline$M$ & $\left\{x_{2}\right\}$ & $1 / 5$ \\
\hline$C$ & $\left\{x_{2}\right\}$ & $1 / 5$ \\
\hline$(A+E) / 2$ & $\left\{x_{2}\right\}$ & $1 / 5$ \\
\hline$(A+M) / 2$ & $\left\{x_{2}\right\}$ & $1 / 5$ \\
\hline$(A+C) / 2$ & $\left\{x_{2}\right\}$ & $1 / 5$ \\
\hline$(M+C) / 2$ & $\left\{x_{2}\right\}$ & $1 / 5$ \\
\hline$(E+M) / 2$ & $\left\{x_{2}\right\}$ & $1 / 5$ \\
\hline$(E+C) / 2$ & $\left\{x_{1}, x_{3}, x_{4}, x_{5}\right\}$ & $4 / 5$ \\
\hline$(A+E+M) / 3$ & $\left\{x_{2}\right\}$ & $1 / 5$ \\
\hline$(A+E+C) / 3$ & $\left\{x_{2}\right\}$ & $1 / 5$ \\
\hline$(A+M+C) / 3$ & $\left\{x_{1}, x_{2}, x_{4}\right\}$ & $3 / 5$ \\
\hline$(E+M+C) / 3$ & $U$ & 1 \\
\hline$(A+M+C+E) / 4$ & $U$ & 1 \\
\hline
\end{tabular}

Then the attribute $A, E, M, C$ are indispensable, and the attributes set $\{A, E\},\{A, C\},\{A, M\},\{E, C\},\{M, C\},\{E, M\}$ are indispensable, and the attribute $\{E, M, C\}$ is dispensable and is reduct, since $\gamma_{\{E, M, C\}}(X)=\gamma_{\mathcal{A}}(X)$, and the attributes $\{A, E, C\},\{A, M, C\},\{A, E, M\}$ are indispensable. Also, we say that $M S, R_{(E+M+C) / 3}$ totally depends on $R_{(A+E+M+C) / 4}$ and $R_{A}, R_{E}, R_{M}, R_{C}, R_{(A+E) / 2,} R_{(A+C) / 2,}$

$R_{(A+M) / 2,} R_{(M+C) / 2,} R_{(E+M) / 2}$. Then the attribute $\{E, M, C\}$ is dispensable and is reduct, since $\gamma_{R_{(E+M+C) / 3}}(X)=\gamma_{M_{S}}(X)$. The attributes $\{A, E, C\},\{A, M, C\},\{A, E, M\}$ are indispensable and $R_{(E+M) / 2}, R_{(A+E+C) / 3}, R_{(A+M+C) / 3}, R_{(A+E+M) / 3}$ rough depends on $R_{(A+E+M+C) / 4}$.

Now we introduce a new method to the reduction of knowledge by using the graph.

Example 3.1. From Example 2.1, we have the graph of attribute set $\mathcal{A}$ as in Figure 3. 


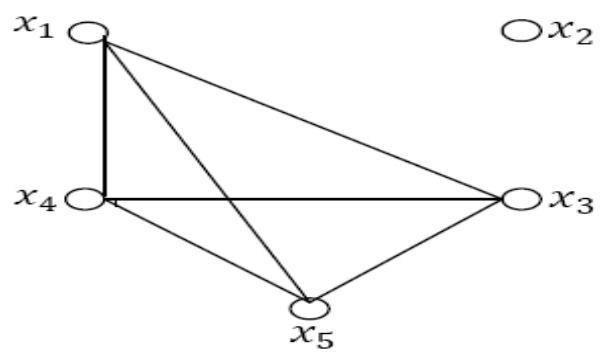

Figure 3

Also, we have the graph of attribute $\mathrm{A}$ as in Figure 4.

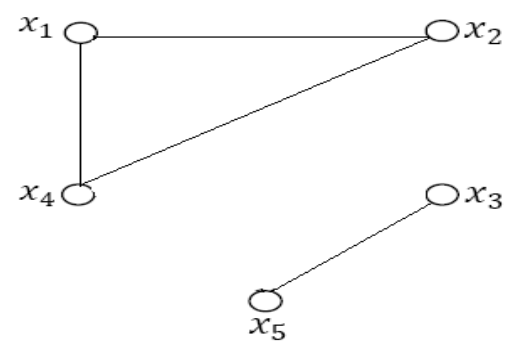

Figure 4

And by the similar way we can drown the graph of all sets of attribute as in Figure 3. and Figure 4. We observing the graph of set $\{E, M, C\}$ is the same of graph of set of attributes $\mathcal{A}=\{A, E, M, C\}$. Then set $\{E, M, C\}$ is the reduct of attributes.

\section{4- Conclusion.}

Pawlak in 1982 defined the concept of approximation space to solve application of computer sciences by using equivalence relation. In [14] the authors introduced the applications of rough sets to graph theory by using neighborhood system. Our work, we used the minimal approximation space to construct a simple undirected graph based on a binary relation. The lower approximation and the upper approximation of subgraph are investigated to compute the rough subgraph. Finally, we used a minimal approximation space and graph theory to reduct the information system and compute the core of knowledge.

\section{Acknowledgment}

The author would like to thank the referees for their helpful comments that improve the presentation of this paper.

\section{References}

1- L. Euler; Solutio problematis ad geometriam situs pertinentis. Comment Academiae Sci. I. Petropolitanae, (1736), 128-140.

2- H.Y. Wang, Q. Huang, C.T. Li and B.Z. Zhe; Graph Theory Algorithm and its Implementation in Matlab, Beihang University press, Beijing, (2010).

3- J.A. Bondy and U.S.R. Murty; Graph Theory with Applications. Elsevier Science Publishing Co. Inc., (1976).

4- J.A. Bondy and U.S.R. Murty; Graph Theory. Springer. Berlin, 2008.

5- R. Diestel; Graph Theory, 3rd ed., Springer-Verlag Heidelberg press, New York, (2005).

6- V. Popa and T. Noiri; On M-continuous functions", Anal. Univ. "Dunarea de Jos" Galati' Ser. Mat. Fiz. Mec. Teor. Fasc. II, 18 (23), (2000), 31-41.

7- Z. Pawlak; Rough sets, International Journal of computer and Information Sciences, Vol. 11, (1982), 341-365.

8- A. M. Kozae, A. Abo-Khadra and M. Shokry; Topology Generated by Graphs. J. of Inst. of Mathematics, Computer Sciences, Vol. 20, No.1 (2009), 49-57.

9- Abd- El Aziz. Radwan, M. shokry Nayle and Ahmed Ibrahem Nazir; New Rough Sets properties on graph theory, Int. Journal Contemp Math Sciences, Vol. 7 No. 25, (2012) ,1217-1232. 
10- J. Stefanowski, On rough set based approaches to induction of decision rules. In: Rough Sets in Data Mining and Knowledge Discovery,Vol1, L. Polkowski , A. Skowron, (eds) Physica Verlag, Heidelberg, (1998), 500-529. On Knowledge Discovery in Data bases, Washington, DC, (1993).

11- M. M. EL-Sharkasy and F. Altwme; Some New Types of Approximations via Minimal Structure. Preprint in Journal of the Egyptian Mathematical Society, 2017.

12- S. Dhanalakshmi, N. Parvathi and R. Arulprasam; A study of Rough Set Approximations for a Complementary Graph, Int. Journal of Scientific, Vol. 6, No.3, (2015), 46-50.

13- Z. Pawlak; Rough set theory and application to data analysis, Cybernetics and system: An International Journal, Vol. 29 (1998), 661-688.

14- J. Chen and J. Li; An application of rough sets to graph theory. Department of mathematics and information Science, Zhangzhou Normal University .PR china, information Science (2012), 114127. 